\title{
Models and valid inequalities to asymmetric skill-based routing problems
}

\author{
Paola Cappanera $\cdot$ Luis Gouveia • \\ Maria Grazia Scutellà
}

Received: 21 May 2012/ Accepted: 14 June 2012/Published online: 11 July 2012

(C) Springer-Verlag + EURO - The Association of European Operational Research Societies 2012

\begin{abstract}
Given a directed network, where a set of skills $S_{j}$ is required to serve each node $j$, and given a set of available technicians, each one having abilities described through a set of skills, we study the problem of defining the tour of each technician in such a way that each service requirement is fulfilled by exactly one technician, and proper skill constraints are satisfied. These constraints state that the service requirement at node $j$ can be operated by any technician having, at least, all the skills in $S_{j}$. Given travelling costs for the arcs of the network, which are technician dependent, we want to determine a set of tours of minimum cost which satisfy the skill constraints. This problem, named asymmetric skill VRP (A-SKVRP), originates from a real application arising in Field Service. Also, it is strongly related to Home Care applications. Starting from the preliminary results in Cappanera et al. (Network optimization. 5th international conference, INOC 2011, Hamburg, June 2011. Lecture notes in computer science, Springer, Berlin, vol 6701, pp 354-364, 2011), where a special case is addressed, we propose a hierarchy of three ILP flow-based models for A-SKVRP, which is based on increasing levels of disaggregation of the flow variables used to prevent subtours. The computational results of a first pool of experiments show that the LP bounds produced by the most disaggregated model are very tight, but at the expense of very large computational times in most cases. This becomes critical as the number of involved nodes and
\end{abstract}

This paper expands and improves upon the LNCS version (Cappanera et al. 2011).

P. Cappanera $(\bowtie)$

Dipartimento di Sistemi e Informatica, Università di Firenze, via S. Marta 3, 50139 Florence, Italy

e-mail: paola.cappanera@unifi.it

\section{Gouveia}

Departamento de Estatística e Investigação Operacional, Centro de Investigação Operacional, Universidade de Lisboa, Lisboa, Portugal

M. G. Scutellà

Dipartimento di Informatica, Università di Pisa, Pisa, Italy 
skills increases. Therefore, in the second part of our study, we enhance the LP bounds obtained with the weaker models by adding, in a cutting plane fashion, cut constraints. As a result of this enhancement, the intermediate model proves to be competitive against the most disaggregated model in computing tight lower bounds, especially as the number of the nodes and of the skills increases. This model combined with the proposed valid inequalities thus qualifies as an efficient tool to address skill-based routing problems arising in application contexts such as Field Service and Home Care.

Keywords Asymmetric VRP · Skill · ILP models · Valid inequalities · Cutting plane $\cdot$ Computational experiments

\section{The problem}

Let $G=(N, A)$ be a (complete) directed network, with $|N|=n$ and $|A|=m$, where node 1 denotes the depot. Let each node $j, j \neq 1$, represent a service requirement, and $S_{j}$ denote the set of the skills required by node $j$ for the associated service call. Let $T$ be the set of available technicians, and let $S_{t}$ denote the set of the skills characterizing the abilities of technician $t$. The skills constrain how the node requirements can be operated; the service requirement at node $j$ can be operated by any technician $t$ possessing all the abilities required by $j$, i.e. $S_{j} \subseteq S_{t}$.

Given non-negative technician-dependent travelling costs $c_{i j}^{t}$ for each $(i, j) \in A$ and technician $t \in T$, we study the problem of defining a set of tours for the technicians, each one starting and ending at node 1 , in such a way that each service requirement is fulfilled by exactly one technician, and the skill constraints are satisfied. This problem is referred to as the asymmetric skill VRP (A-SKVRP). It has been introduced in Cappanera et al. (2011) for the special case where a skill $s_{j}$ is associated with each node $j$, a skill $s_{t}$ is associated with each technician $t$, and there is a gradation of the skills such that $j$ can be visited by $t$ only if $s_{t} \geq s_{j}$. A-SKVRP originates from a real application concerning the dispatch of technicians to customer service requests in after-sales service management, referred to as Field Service (Agnihothri and Mishra 2004; Rapaccini et al. 2008). Also, it is strongly related to Home Care applications like Home Care Scheduling problems, where coordinators have to assign the care of every client to an operator by considering his workload, the particular skills that the operator should have in order to satisfy the client needs and the travel distance of the operator for reaching client home. See Borsani et al. (2006) for an example of an Integer Linear Programming (ILP) model developed to support human resource short-term planning in Home Care Scheduling where, however, routing aspects are not addressed.

Although the asymmetric skill VRP bears some similarities to the site-dependent VRP (SDVRP), introduced by Nag et al. (1988), where a node $j$ can only be served by a subset of the vehicles (or technicians, in our terminology), observe that A-SKVRP is not a special case of SDVRP. In SDVRP the routing costs are vehicle independent and represented by a symmetric edge cost matrix (Baldacci et al. 2010c). See Baldacci et al. (2010a, b) for exact approaches to SDVRP, and Cordeau 
and Laporte (2001) for an example of Tabu Search approach for the SDVRP with time windows. On the other hand, in A-SKVRP the costs are technician dependent and represented by an asymmetric cost matrix.

Also observe that, if we restrict the attention to undirected graphs (and so to symmetric edge cost matrices), then the A-SKVRP can be formulated as a special case of the Multi-Depot VRP (MDVRP), which is more general than SDVRP. In fact, MDVRP is an extension of the Capacitated VRP (CVRP) where a node can be served by an unlimited fleet of identical vehicles of capacity $Q$, located at $p$ depots, inter-depot routes are not allowed, and costs may vary depending on the depot (Baldacci et al. 2010c). To model the symmetric skill VRP in terms of MDVRP, just introduce a depot $d_{t}$ of capacity $n-1$ for each technician $t$ [i.e. introduce a copy of the original depot (node 1) for each technician], and associate with $d_{t}$ the arc costs which depend on $t$.

In any case it is important to emphasize that A-SKVRP is introduced and studied in the more general scenario of asymmetric graphs, and thus it is a special case of the asymmetric MDVRP which however, to the best of our knowledge, has never been investigated in the literature so far.

Starting from the preliminary results in Cappanera et al. (2011), in this paper, we propose a hierarchy of ILP flow-based models for A-SKVRP, which is based on increasing levels of disaggregation of the flow variables used to prevent subtours. Flow-based models are a well-known modeling tool to formulate VRP problems, as shown in Gavish and Graves (1979) and Letchford and Salazar-Gonzalez (2006). Specifically, in "ILP models for the Asymmetric Skill VRP" we present three ILP models such that the Linear Programming (LP) bound of the most disaggregated model is greater than or equal to the one associated with the intermediate model, which in turn is greater than or equal to the LP bound of the less disaggregated model. We investigate the behavior of the three models by performing a first pool of experiments on randomly generated instances characterized by a gradation of three skills, such that node $j$ can be visited by technician $t$ only if $s_{t} \geq s_{j}$. The computational results, discussed in "Model comparison", provide evidence that the LP bounds produced by the most disaggregated model are very tight. This model thus qualifies as a strong model to solve A-SKVRP instances. However, such a model may be difficult to use, since the computational time needed to solve its LP relaxation tends to increase with the number of the nodes. In addition, it also increases with the number of the skills, and this becomes critical in the abovementioned application contexts, i.e. Field Service and Home Care, where often the number of the skills is larger than 3 , which is the case addressed in the first pool of experiments. Indeed, the size of the most disaggregated model increases with the number of skills and technicians, both in terms of number of variables and constraints.

Due to the considerations above, in the second part of our study we investigate the strength of the most disaggregated model in more depth, by observing that its LP relaxation implies an exponential sized set of cut constraints. Then, in order to achieve a compromise between strength of the LP bound and computational effort required to compute it, we select a suitable subset from this large class of cut constraints, and use it to enhance the LP bounds obtained with the weaker models. 
This idea of projecting some constraints satisfied by the solutions of an extended formulation into more compact formulations is a well-known modeling technique to improve the LP relaxation of ILP formulations, see for example Letchford and Salazar-Gonzalez (2006). The projected cuts are described in "Special cut constraints", whereas the computational results conducted to assess the impact of the proposed valid inequalities are discussed in "Assessing the impact of valid inequalities". These results show that the intermediate model becomes a better alternative than the most disaggregated model after the cut addition. We then study an additional set of cut constraints, of exponential size, and further enhance the intermediate model via a cutting plane approach. The proposed approach, presented in "Two-cycle inequalities and related cutting plane approach", proves to be very effective as the number of nodes and skills increases, thus promoting the intermediate model and the proposed valid inequalities as an efficient tool to address skill-based routing problems arising in application contexts such as Field Service and Home Care. This is reported in "Assessing the impact of the cutting plane approach". Finally, "Conclusions" propose suggestions for future research.

We conclude this section by pointing out that, to the best of our knowledge, no formulations and exact approaches have been proposed in the literature to the asymmetric MDVRP. Therefore, no comparison can be performed between the formulations proposed in this paper for A-SKVRP (and the related computational results) and alternative approaches from the literature for the more general asymmetric MDVRP. A comparison might be performed only between the symmetric skill VRP and MDVRP. Anyway, although very efficient exact approaches have been proposed for the symmetric VRP, and in particular to MDVRP, all based on set partitioning formulations, indeed the best state-of-the-art exact code for symmetric VRPs, described in Baldacci et al. (2010c), may not be so efficient to address the symmetric skill VRP instances, due to the intrinsic uncapacitated nature of skill VRP. ${ }^{1}$

\section{ILP models for the asymmetric skill VRP}

In Cappanera et al. (2011) three ILP models have been proposed in order to formulate the asymmetric skill VRP when a gradation of the skills is defined. Here, the models are generalized to the case where arbitrary subsets of skills characterize nodes as well as technicians. The three models share the design variables and some sets of constraints which, as classical in the VRP literature, are used to define the tours of the technicians. The objective function, to be minimized, is also common to the three models. For each $(i, j) \in A$ and $t \in T$ such that $\left(S_{i} \cup S_{j}\right) \subseteq S_{t}$, let:

$$
x_{i j}^{t}=\left\{\begin{array}{cc}
1 & \text { if }(i, j) \text { belongs to the tour of technician } t \\
0 & \text { otherwise }
\end{array}\right.
$$

Note that condition $\left(S_{i} \cup S_{j}\right) \subseteq S_{t}$, for each arc $(i, j)$ and technician $t$, allows one to incorporate the skill constraints directly into the variable definition. The design

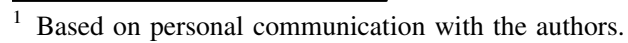


variables must satisfy constraints (1), (2) and (3). In particular, the first set of constraints guarantees that each client is visited once, while the second set guarantees the skill consistency along the tour of technician $t$, i.e. if a properly skilled technician $t$ enters a node $j$, then $t$ leaves $j$.

$$
\begin{gathered}
\sum_{i \in N} \sum_{t:\left(S_{i} \cup S_{j}\right) \subseteq S_{t}} x_{i j}^{t}=1 \quad j=2, \ldots, n \\
\sum_{i \in N: S_{i} \subseteq S_{t}} x_{i j}^{t}=\sum_{i \in N: S_{i} \subseteq S_{t}} x_{j i}^{t} \quad j=2, \ldots, n, t: S_{j} \subseteq S_{t} \\
x_{i j}^{t} \in\{0,1\} \quad(i, j) \in A, t:\left(S_{i} \cup S_{j}\right) \subseteq S_{t}
\end{gathered}
$$

The objective function, to be minimized, defines the overall routing cost:

$$
\sum_{(i, j) \in A} \sum_{t:\left(S_{i} \cup S_{j}\right) \subseteq S_{t}} c_{i j}^{t} x_{i j}^{t}
$$

The ILP models differ in the way they prevent subtours, that is tours which are disconnected from the depot. The proposed models use auxiliary flow variables and standard flow conservation constraints, plus linking constraints to relate the flow variables to the design variables, on increasing levels of variable and constraint disaggregation. Skill information is suitably added to the linking constraints, depending on the considered level of disaggregation. Let us discuss these flow model components in more detail.

The aggregated model

The first model, called Agg, prevents subtours using an auxiliary flow variable $y_{i j}$ for each $(i, j) \in A$, that indicates the amount of flow pushed along $(i, j)$ [or, equivalently, the number of clients to be visited after traversing the arc $(i, j)$, included node $j]$. This is typical in aggregated flow (or single commodity flow) formulations for vehicle routing problems. See Gavish and Graves (1979) for pioneering flow-based formulations in the context of VRP.

Using these flow variables and the design variables $x_{i j}^{t}$, model Agg comprises the following constraints in addition to (1)-(3):

$$
\begin{aligned}
& \sum_{(l, j) \in A} y_{l j}=n-1 \\
& \sum_{(i, j) \in A} y_{i j}-\sum_{(j, i) \in A} y_{j i}=1 \quad j \in N \backslash\{1\} \\
& y_{i j} \leq(n-1) \sum_{t:\left(S_{i} \cup S_{j}\right) \subseteq S_{t}} x_{i j}^{t} \quad(i, j) \in A \\
& y_{i j} \geq 0 \quad(i, j) \in A
\end{aligned}
$$

As specified before, the skill-based routing is determined by minimizing function (4). The first group of constraints states that $n-1$ units of flow leave the depot, while the second group guarantees that each client receives one unit of flow. The third group is formed by the constraints linking the flow variables to the design variables. 
The destination disaggregated model

The second model is obtained from Agg by performing a disaggregation of the flow variables $y_{i j}$ by destination. This way of strengthening the linear programming relaxation of single commodity flow models is a well-known technique (to the best of our knowledge, this technique was first suggested by Rardin and Choe (1979). Specifically, replace the flow variables $y_{i j}$ by the disaggregated flow variables $y_{i j}^{k}, k \in N \backslash\{1\}$, that specify the amount of flow traversing the $\operatorname{arc}(i, j)$ that is destined to node $k$. In particular, the variables $y_{i j}^{k}$ are defined for $j \neq 1$ and $i \neq k$.

Using these disaggregated flow variables and the design variables $x_{i j}^{t}$, we obtain a new model, referred to as DestDis, which includes the following constraints in addition to the common block (1)-(3):

$$
\begin{gathered}
\sum_{(i, j) \in A: i \neq k, j \neq 1} y_{i j}^{k}-\sum_{(j, i) \in A: i \neq 1, j \neq k} y_{j i}^{k}=\left\{\begin{array}{cl}
1 & \text { if } i=1 \\
0 & \text { if } i \neq 1, k \quad i \in N, k=2, \ldots, n \\
-1 & \text { if } i=k
\end{array}\right. \\
y_{i j}^{k} \leq \sum_{t:\left(S_{i} \cup S_{j} \cup S_{k}\right) \subseteq S_{t}} x_{i j}^{t} \quad(i, j) \in A, k \in N: k \neq 1, i, j \neq 1 . \\
y_{i j}^{k} \geq 0 \quad(i, j) \in A: j \neq 1, k \neq 1, i
\end{gathered}
$$

Again, the objective function is given by (4).

In model DestDis, the flow-based constraints state that one unit of flow must be sent from the depot to each client or, equivalently, that the solution must contain a path from the depot to node $k$, for each $k \in N \backslash\{1\}$. Thus, the flow variables $y_{i j}^{k}$ can be reinterpreted as indicating whether the arc $(i, j)$ is in the path from the depot to node $k$.

Observe that model DestDis is more than a straightforward multicommodity reformulation of model Agg. In fact, a straightforward disaggregation would lead to the weaker linking constraints $y_{i j}^{k} \leq \sum_{t:\left(S_{i} \cup S_{j}\right) \subseteq S_{t}} x_{i j}^{t},(i, j) \in A, k \in V, k \neq 1, i, j \neq 1$. On the other hand, the flow variable disaggregation, together with the skill definition, allows one to enhance the linking constraints, leading to the strengthened form (5). This suggests that the LP bound of DestDis should be substantially tighter than the one returned by $\mathrm{Agg}$ (it is easy to see that it is always greater than or equal to the LP bound of model $\mathrm{Agg}$ ).

The destination-technician disaggregated model

The third model combines disaggregation by destination with disaggregation by technician. In fact, by performing a further step of disaggregation, the flow variables $y_{i j}^{k}, k \in N \backslash\{1\}$, which characterize model DestDis, are replaced by the flow variables $y_{i j}^{k t}$, with $j \neq 1, i \neq k$ and $t:\left(S_{i} \cup S_{j} \cup S_{k}\right) \subseteq S_{t}$. Each variable $y_{i j}^{k t}$ indicates whether the arc $(i, j)$ belongs to the path from the depot to node $k$, and whether it is traversed by technician $t$. The model uses additional auxiliary variables $w^{k t}, k=2, \ldots, n, t: S_{k} \subseteq S_{t}$, indicating whether node $k$ is served by technician $t$. 
Using the variables $y_{i j}^{k t}$ and $w^{k t}$, the model, hereafter named DestTechDis, comprises the following constraints in addition to (1)-(3), under the minimization stated by (4):

$$
\sum_{\substack{(i, j) \in A: i \neq k, j \neq 1,\left(S_{i} \cup S_{j}\right) \subseteq S_{t}}} y_{i j}^{k t}-\sum_{\substack{(j, i) \in A: i \neq 1, j \neq k,\left(S_{i} \cup S_{j}\right) \subseteq S_{t}}} y_{j i}^{k t}=\left\{\begin{array}{cl}
w^{k t} & \text { if } i=1 \\
0 & \text { if } i \neq 1, k \quad i \in N, k=2, \ldots n, t: S_{k} \subseteq S_{t} \\
-w^{k t} & \text { if } i=k
\end{array}\right.
$$

$$
\begin{gathered}
\sum_{t: S_{k} \subseteq S_{t}} w^{k t}=1 \quad k=2, \ldots, n \\
y_{i j}^{k t} \leq x_{i j}^{t} \quad(i, j) \in A, \quad k \in N: j \neq 1, k \neq 1, i, \\
t:\left(S_{i} \cup S_{j} \cup S_{k}\right) \subseteq S_{t} \\
w^{k t}=\sum_{j \in N: S_{j} \subseteq S_{t}} x_{j k}^{t} \quad k=2, \ldots, n, t: S_{k} \subseteq S_{t} \\
w^{k t} \in\{0,1\} \quad k=2, \ldots, n, t: S_{k} \subseteq S_{t} \\
y_{i j}^{k t} \geq 0 \quad(i, j) \in A: j \neq 1, k \neq 1, i, \quad t:\left(S_{i} \cup S_{j} \cup S_{k}\right) \subseteq S_{t}
\end{gathered}
$$

The flow conservation constraints (6) state that the variables $y_{i j}^{k t}$ define a path from the depot to node $k$, if $k$ is served by technician $t$ (i.e. $w^{k t}=1$ ). Note that, if we relate these flow variables to the flow variables of model DestDis, according to $\sum_{t} y_{i j}^{k t}=y_{i j}^{k},(i, j) \in A: j \neq 1, k \neq 1, i$, then the linking constraints (8) can be viewed as a disaggregated form of the linking constraints in model DestDis. This shows that the LP bound of DestTechDis is never worse than the LP bound of DestDis, and also suggests that the LP bound of DestTechDis can be tighter than the one returned by DestDis.

Equalities (9) indicate that the variables $w^{k t}$ can be eliminated from the model. However, they are maintained to make the flow conservation constraints easier to interpret. Note also that of the three sets of equalities, (1), (7) and (9), two sets imply the other, and so one set can be removed. This is easily seen in the case of (1) or (7) being redundant. For the other case, consider the following result:

Lemma 1 The constraints $w^{k t}=\sum_{j \in N: S_{j} \subseteq S_{t}} x_{j k}^{t}, k=2, \ldots, n, t: S_{k} \subseteq S_{t}$, are redundant in the LP relaxation of model DestTechDis.

Proof Remove constraints (9) from model DestTechDis. Then observe that the LP of the resulting, reduced, model implies the constraints $w^{k t} \leq \sum_{j \in N: S_{j} \subseteq S_{t}} x_{j k}^{t}, k=$ $2, \ldots, n, t: S_{k} \subseteq S_{t}$. This follows from (6) and (8).

Then, by adding these inequalities over $t$, and combining with (1) and (7), we conclude that these inequalities cannot be strict. Therefore, constraints (9) are implied by the LP relaxation of the reduced model or, equivalently, they are redundant in the LP relaxation of DestTechDis. The thesis follows.

A hierarchy of ILP flow-based models for A-SKVRP has thus been presented, which is based on increasing levels of disaggregation of the flow variables. As already observed, the LP bound of the most disaggregated model is greater than or 
equal to the one associated with the intermediate model DestDis, which, in turn, is greater than or equal to the LP bound of $\mathrm{Agg}$. We have investigated this relationship in more detail through a first pool of experiments reported in "Model comparison". The main conclusion is that model DestTechDis produces very tight LP bounds. However, it may be difficult to use in practice, since the computational time needed to solve its LP relaxation is very large in most cases. Therefore, as already outlined, we have analyzed more closely the relation between the LP relaxation of the models DestTechDis and DestDis and this is the topic of the next section.

\section{Valid inequalities and cutting plane approach}

In this section, we discuss the strength of the LP relaxation of the model DestTechDis. We present a large subset of cut-set inequalities, on the variables $x_{i j}^{t}$, which are implied by the LP relaxation of this model. Then, based on the analysis of the LP solutions of the weaker models, we select a subset of the new inequalities in order to improve the LP relaxation of the weaker models, as reported in "Experimental campaign".

Strength of model DestTechDis

From the maximum flow-minimum cut theorem (see, e.g. Ahuja et al. 1993), it is easy to show that the LP relaxation of DestTechDis implies the following exponential sized set of cut constraints:

$$
\sum_{i \in N \backslash S, j \in S:\left(S_{i} \cup S_{j}\right) \subseteq S_{t}} x_{i j}^{t} \geq \sum_{j \in N: S_{j} \subseteq S_{t}} x_{j k}^{t} \quad S \subseteq N \backslash\{1\}, k \in S, t \in T: S_{k} \subseteq S_{t} .
$$

Based on this observation, we have selected a suitable subset from the large class of cut constraints (10), and use it to enhance the weaker models Agg and DestDis.

Specifically, by looking at the subgraphs induced by the variables $x_{i j}^{t}$ having a positive value in the LP solution of models Agg and DestDis, for each $t$, we observed the following facts:

1. the LP solution contains many two cycles (i.e. cycle of type $(i-j-i)$ ) or composition of two cycles in the subgraphs associated with technicians $t$ not used in the solution (i.e. $x_{1 j}^{t}=0$ for all nodes $j$ ).

2. the LP solution contains two cycles (or again composition of two cycles) also in the subgraphs of technicians used, i.e. connected to the depot.

Special cases of constraints (10) have therefore been investigated in order to eliminate these two-cycle structures, and to enhance the weaker models going toward the quality of the most disaggregated model.

Special cut constraints

In the first part of the analysis devoted to the enhancement of the weaker models, the following special cut constraints, of polynomial size, have been added to the LP relaxation of Agg and DestDis: 


$$
\begin{gathered}
\sum_{j \in N \backslash\{1\}: S_{j} \subseteq S_{t}} x_{1 j}^{t} \geq \sum_{(j, i): S_{j} \subseteq S_{t}} x_{j i}^{t} \quad t \in T, i \neq 1: S_{i} \subseteq S_{t} \\
x_{i k}^{t} \leq \sum_{(j, i): j \neq k, S_{j} \subseteq S_{t}} x_{j i}^{t} \quad t \in T, i, k \neq 1:\left(S_{i} \cup S_{k}\right) \subseteq S_{t} .
\end{gathered}
$$

Constraints (11) guarantee that, if technician $t$ is not used, then none of the design variables relative to $t$ can be greater than zero. Constraints (12) state that, if the arc $(i, k)$ belongs to the solution, then the predecessor of node $i$ must be other than $k$. Hereafter these two sets of valid inequalities will be referred to as TC1 and TC2, respectively, where TC stands for two cycles. Agg and DestDis augmented with TC1 and TC2 will be referred to as StrongAgg and StrongDestDis, respectively.

Observe that (11) are special cut constraints of type (10) obtained by choosing $S=\{1\}$. These valid inequalities allow one to eliminate the two cycles of the first type. Also, (12) are special cut constraints, as shown below.

Lemma 2 (12) are special cut constraints of type (10).

Proof Consider the following cut constraints of type (10), obtained by choosing $S=i, k$ :

$$
\sum_{(j, k): j \neq i, S_{j} \subseteq S_{t}} x_{j k}^{t}+\sum_{(j, i): j \neq k, S_{j} \subseteq S_{t}} x_{j i}^{t} \geq \sum_{(j, k): S_{j} \subseteq S_{t}} x_{j k}^{t} .
$$

(13) are equivalent to:

$$
\sum_{(j, i): j \neq k, S_{j} \subseteq S_{t}} x_{j i}^{t} \geq x_{i k}^{t}
$$

The thesis follows.

Two-cycle inequalities and related cutting plane approach

To further enhance model DestDis, which proved to be very competitive after the additions of cuts TC1 and TC2 (see "Assessing the impact of valid inequalities"), another set of cut constraints has been addressed in order to eliminate the two cycles which still arise in the LP solutions. These are cut constraints of type (10) where $S$ is a set of nodes which induces a two-cycle [i.e. a cycle of type $(i-j-i)$ ] or a composition of two cycles in the solution of the LP relaxation of StrongDestDis. These cut constraints will be referred to as two-cycle inequalities. Since the cardinality of this set may be exponential with respect to the input size, a heuristic separation procedure has been implemented to separate inequalities from this subset, and a cutting plane approach has been designed based on this separation procedure. For each technician $t$, the procedure visits the graph starting from the depot. It stores in $S$ a potential candidate node $k$, and a subset of nodes $i$ and $j$, reachable from $k$, such that both $x_{i j}^{t}>0$ and $x_{j i}^{t}>0$. The key idea is to identify a composition of two cycles, that includes $k$, in the subgraph associated with technician $t$. The constructed set $S$ and the corresponding $k$ are returned by the separation procedure only if $|S| \geq 3$ (since the valid inequalities TC1 and TC2 are already included into the model), and if the total sum of the variables $x_{i j}^{t}$ within 
$S$, that is for $i$ and $j$ belonging to $S$, is "sufficiently high", i.e. greater than or equal to $|S| / 2$. The last condition guarantees that, in the subsets $S$ returned by the heuristic, the majority of the nodes are connected through links which are inner to the subset. Our testing showed that this heuristic proved to be very efficient in separating violated two-cycle inequalities. Each iteration of the cutting plane approach consists in performing the separation procedure described above, and adding to the model the packet of violated two-cycle inequalities discovered by this procedure. In other words, several violated inequalities may be added all in each iteration of the proposed approach.

Observe that in the skill VRP scenario where a single skill $s_{j}$ is associated with each node $j$, a single skill $s_{t}$ is associated with each technician $t$, and a gradation of the skills is defined such that a node $j$ can be visited by a technician $t$ only if $s_{t} \geq s_{j}$, then the A-SKVRP models, and the related valid inequalities, can be derived from the ILP formulations and the valid inequalities presented above by replacing the operator $\subseteq$ by $\leq$ in the stated skill constraints. For example, $j, t: S_{j} \subseteq S_{t}$ becomes $j, t: s_{j} \leq s_{t}$. This is the scenario addressed in the experimental campaign.

\section{Experimental campaign}

The main achievements that are shown, respectively, in the next three subsections are the following: (a) model DestTechDis has a very strong LP relaxation; unfortunately, the computational time required to solve the LP relaxation becomes unacceptable on large instances that arise from real application contexts; (b) the weaker models Agg and DestDis greatly benefit from the introduction of a subset of valid inequalities that are implied by model DestTechDis; in particular, the gap of the intermediate model DestDis becomes very tight after the introduction of some of these constraints, suggesting that DestDis is a good tradeoff between quality of the bounds and computational effort required to compute them; (c) a cutting plane approach based on model DestDis with an additional set of cut inequalities, when applied to large instances, is able to provide solutions very close (within $2 \%$ in most cases) to the ones obtained by the stronger model DestTechDis, in much shorter computational times.

All the models and the cutting plane approach have been implemented via AMPL using the solver Cplex 12.1 on a AMD Athlon $(\mathrm{tm}) 64 \times 2$ Dual Core Processor $4200+$ (CPU MHz 2211.186). In the following the computational times are expressed in seconds of CPU time.

Model comparison

Here, we present the results of the first pool of experiments on a suite of randomly generated instances characterized by three skills. As indicated before, a skill $s_{j}$ is associated with each node $j$, a skill $s_{t}$ is associated with each technician $t$, and a gradation of the skills is defined such that a node $j$ can be visited by a technician $t$ only if $s_{t} \geq s_{j}$. Symmetric as well as asymmetric instances compose this suite of 
A-SKVRP instances. The experiments aim at comparing the ILP models presented in "ILP models for the Asymmetric Skill VRP" both in terms of quality of the returned LP bounds and also in terms of the required computational times.

\section{A-SKVRP instances: scenario with three skills}

We used two test sets originally suggested for the VRP, with the data relative to the skills. Specifically, the VRP instances of the first test set are characterized by the number of nodes (either 20 or 40) and by the type ( 4 different choices). There are two types of Euclidean instances referred to as $t c$ and $t e$ according to the position of the depot in the grid. In the $t c$ instances the depot is located on a corner of the grid, while in the te instances the depot is located in the centre of the grid. In addition, there are two types of random instances: tra denotes asymmetric instances and trs denotes the symmetric ones. Five instances are available for each given number of nodes and type, thus summing up to a total of $40(2 \times 4 \times 5)$ instances. The VRP instances $t c$ and te were created for the computational testing reported in Gouveia (1995), and since then they have been used in the computational testing reported in many other papers concerning trees, for instance Gouveia et al. (2011). These instances have also been used for the results reported in the paper on VRP (Godinho et al. 2009). The VRP instances tra and trs have been generated for the experiments in Godinho et al. (2009).

In order to generate the first group of A-SKVRP instances we used instance number one (over the five available) for each type and for each given number of nodes, thus summing up to 8 original instances over 40 .

The VRP instances of the second test set are asymmetric CVRP instances proposed in Fischetti et al. (1994) and available at http://www.or.deis.unibo. it/research.html. They are characterized by a number of nodes in $\{34,36,39,45,48,56,65,71\}$ and by three vehicles. In order to generate the second group of A-SKVRP instances we used the larger instances in this test set, i.e. A045, A048, A056, A065 and A071.

In the generated A-SKVRP instances there is a one-to-one correspondence between skills and technicians, i.e. we have a technician for each skill. In this pool of experiments we set both the number of technicians and the number of skills equal to 3 (observe that this is the number of vehicles characterizing the asymmetric instances in Fischetti et al. 1994), where skill 1 denotes the lowest skill and skill 3 is the highest (w.l.o.g. the depot is assumed to have skill 1).

For each original instance of the first test set we generated 18 A-SKVRP instances according to the pattern used to assign a skill to the nodes. The patterns used are the following:

50-10-40 (for a total of 6 permutations)

50-20-30 (for a total of 6 permutations)

40-40-20 (for a total of 3 permutations)

30-30-40 (for a total of 3 permutations)

where the numbers indicate the percentage of nodes requiring a specific skill. As an example, pattern 50-10-40 is used to denote possible scenarios where $50 \%$ of nodes 
requires a certain skill, which can be skill 1,2 or $3,40 \%$ of nodes requires another skill, and $10 \%$ of nodes requires a third skill, thus summing up to 6 combinations.

For each original instance of the second test set we generated six A-SKVRP instances according to the following patterns:

$50-40-10$
$40-50-10$
$50-30-20$
$30-50-20$
$40-40-20$
$40-30-30$

In contrast to the original VRP instances used, in the A-SKVRP instances the costs depend on the arc as well as on the technician. We assumed that the cost of an arc $(i, j)$ is increasing with the technician skills, i.e. the more a technician is qualified higher the cost. The formula used to generate the costs is the following:

$$
c_{i j}^{t}=c_{i j} \delta t,
$$

where $c_{i j}$ represents the cost of arc $(i, j)$ in the original instances, while the factor $\delta$ has been set equal to 2 . Observe that these costs satisfy the triangular property for each technician when the original costs do. Trivially if $c_{i j} \leq c_{i h}+c_{h j}$, the inequality $c_{i j} \delta k \leq\left(c_{i h}+c_{h j}\right) \delta k$ holds true. Under the hypothesis of costs not satisfying the triangular property, the proposed models do not assure that, for a given technician, the schedule is composed of a single tour, i.e. it might be cheaper for the technician come back to the depot more than once.

\section{Computational results}

We have performed our computational experiments with the instances of the two groups. In Tables 1, 2 and 4, a comparison of the models Agg, DestDis and DestTechDis on the larger instances of the first group, i.e. the ones with 40 nodes, is given in terms of average time required to solve the continuous relaxation and in terms of percentage gap of the LP bound with respect either to the optimum value when available or to the best integer solution value. Model Agg is used to get the optimum value or an upper bound to it. For the aggregated model the computational time required to get the optimum is also reported: a time limit of $6 \mathrm{~h}$ is fixed and when such a limit is exceeded the value of the best integer solution is returned. Since the computational time was not an issue for the instances with 20 nodes, which were solved quite easily via model $\mathrm{Agg}$, the computational results related to such a subset of instances are omitted. Similarly, in Tables 3 and 5 a comparison of the models Agg, DestDis and DestTechDis is given for the instances of the second group. Again, model Agg is used to get the optimum value or an upper bound to it. Due to their very large size, a time limit of $12 \mathrm{~h}$ has been fixed for the instances belonging to the families $A 065$ and A071, i.e. the A-SKVRP instances with 65 and 71 nodes. 
Table 1 Comparison in terms of optimality gap: first group (40 nodes-tc-te)

\begin{tabular}{|c|c|c|c|c|}
\hline Instance & AggIPTime & Agg & DestDis & DestTechDis \\
\hline tc40-1-20-4-16.dat & $21,741.87$ & 30.24 & 6.03 & 1.92 \\
\hline tc40-1-20-16-4.dat & $21,699.26$ & 39.77 & 11.49 & 3.23 \\
\hline tc40-1-4-20-16.dat & $2,865.86$ & 22.73 & 2.52 & 0.30 \\
\hline tc40-1-16-20-4.dat & $21,691.04$ & 39.73 & 10.64 & 4.41 \\
\hline tc40-1-4-16-20.dat & 742.10 & 20.34 & 1.94 & 0.00 \\
\hline tc40-1-16-4-20.dat & $4,907.78$ & 26.43 & 4.24 & 0.73 \\
\hline tc40-1-20-12-8.dat & $21,718.93$ & 35.23 & 11.83 & 3.52 \\
\hline tc40-1-20-8-12.dat & $19,093.48$ & 33.86 & 4.00 & 0.18 \\
\hline tc40-1-8-20-12.dat & $21,653.45$ & 30.04 & 4.55 & 0.59 \\
\hline tc40-1-12-20-8.dat & $21,694.86$ & 31.22 & 8.42 & 1.79 \\
\hline tc40-1-8-12-20.dat & 175.17 & 21.10 & 1.85 & 0.00 \\
\hline tc40-1-12-8-20.dat & $4,476.51$ & 29.63 & 5.70 & 1.22 \\
\hline tc40-1-16-16-8.dat & $21,695.64$ & 35.99 & 10.17 & 4.36 \\
\hline tc40-1-8-16-16.dat & 985.21 & 24.32 & 1.91 & 0.00 \\
\hline tc40-1-16-8-16.dat & $3,992.99$ & 28.66 & 3.48 & 0.82 \\
\hline tc40-1-16-12-12.dat & $3,469.94$ & 29.41 & 4.37 & 0.13 \\
\hline tc40-1-12-16-12.dat & $21,742.12$ & 30.76 & 6.01 & 0.49 \\
\hline tc40-1-12-12-16.dat & $3,184.70$ & 24.14 & 2.08 & 0.00 \\
\hline Avg tc $40-1$ & $12,085.05$ & 29.64 & 5.62 & 1.32 \\
\hline te $40-1-20-4-16 . d a t$ & $1,192.86$ & 23.93 & 5.24 & 0.38 \\
\hline te $40-1-20-16-4 . d a t$ & $21,709.62$ & 38.92 & 16.87 & 8.85 \\
\hline te $40-1-4-20-16 . d a t$ & 20.46 & 17.67 & 5.30 & 0.00 \\
\hline te $40-1-16-20-4 . d a t$ & $21,709.06$ & 39.44 & 15.10 & 8.78 \\
\hline te $40-1-4-16-20 . d a t$ & 7.31 & 15.09 & 2.66 & 0.00 \\
\hline te $40-1-16-4-20 . d a t$ & $2,611.68$ & 20.07 & 4.40 & 0.28 \\
\hline te $40-1-20-12-8 . d a t$ & $21,701.03$ & 32.50 & 11.66 & 4.33 \\
\hline te $40-1-20-8-12 . d a t$ & $21,721.15$ & 28.63 & 6.52 & 0.00 \\
\hline te $40-1-8-20-12 . d a t$ & $21,704.39$ & 33.11 & 15.77 & 9.44 \\
\hline te $40-1-12-20-8 . d a t$ & $21,697.92$ & 32.52 & 12.88 & 3.79 \\
\hline te $40-1-8-12-20 . d a t$ & 181.85 & 15.72 & 5.44 & 0.00 \\
\hline te $40-1-12-8-20 . d a t$ & 154.84 & 16.49 & 3.91 & 0.00 \\
\hline te $40-1-16-16-8 . d a t$ & $21,739.62$ & 29.04 & 11.26 & 2.66 \\
\hline te $40-1-8-16-16 . d a t$ & $2,729.12$ & 20.53 & 6.23 & 0.42 \\
\hline te $40-1-16-8-16 . d a t$ & $21,757.85$ & 25.56 & 11.89 & 2.09 \\
\hline te40-1-16-12-12.dat & $6,681.37$ & 24.02 & 4.04 & 0.00 \\
\hline te $40-1-12-16-12 . d a t$ & $21,731.71$ & 31.78 & 11.54 & 2.49 \\
\hline te40-1-12-12-16.dat & $9,919.81$ & 23.55 & 9.28 & 0.30 \\
\hline Avg te $40-1$ & $12,165.09$ & 26.03 & 8.89 & 2.43 \\
\hline
\end{tabular}

It appears that, in the first group of instances, computing the optimum integer solution is computational expensive for the instances of type $t c$ and te (see the average computational times reported under AggIP in Table 4), whereas the ones of 
type tra are efficiently solved to optimality via model Agg. The instances of type trs show an intermediate behavior. Also note that for $t c$ and te instances the time limit is reached, respectively, 8 and 9 times over 18, whereas for tra and trs instances the optimum is always returned significantly earlier than the time limit. In all cases, the enhancement produced by increasing the level of disaggregation leads to significant improvements of the LP bounds (see the average gaps in bold in Tables 1,2). This trend is particularly evident for the most difficult instances of the group, i.e. the ones of type $t c$ and $t e$, where the most disaggregated model DestTechDis provides an average gap of 1.32 for $t c$ family and of 2.43 for te family, while the average gaps provided by model Agg are, respectively, 29.64 and 26.03 for the two families. Note that DestTechDis is able to find a solution of optimal value in 20 cases over 72 with a bigger frequency on the te and the trs instances (respectively, 6 and 7 times over 18). Clearly, the required computational times become higher when the level of disaggregation increases, reaching an average time of $855.29 \mathrm{~s}$ with DestTechDis for the te instances. Model DestDis shows an intermediate behavior for all the tested instances, in terms of gap and often also in terms of computational time. In fact, the gaps provided by DestDis are much better than the ones related to model Agg, although not tight as the ones computed by DestTechDis.

If we now consider the second group of instances, we notice that these large asymmetric instances are very hard to solve to optimality. In fact, in only 10 cases over 30 the time limit is not exceeded. Observe, in particular, that the time limit of $12 \mathrm{~h}$ set to solve to optimality the A-SKVRP instances with 65 and 71 nodes is always over. Also for this group of instances the enhancement of the level of disaggregation improves the LP bounds in a significant way (see the average gaps in bold in Table 3). In particular, the most disaggregated model DestTechDis provides a very good average gap that ranges from the minimum 1.92 (A045 family) to the maximum 6.10 (A065 family), while the average gaps provided by model Agg range from 17.60 (A045 family) to 23.30 (A056 family). However, also in this case the required computational times become prohibitive when the level of disaggregation increases, reaching an average time of 15537.47 s with DestTechDis for the larger instances with 71 nodes. As before, model DestDis has an intermediate behavior for all the tested instances of the second group, in terms of gap and also in terms of computational time. In particular, the gaps provided by DestDis are much better than the ones related to model Agg, although not tight as the ones computed by DestTechDis. Observe, however, that for the larger instances, i.e. the ones with 56, 65 and 71 nodes, the average gaps provided by DestDis are at most twice the gaps returned via model DestTechDis (see the average gaps reported in bold in Table 3).

As a summary, in both groups of instances the LP bounds produced by DestTechDis are very tight. However, DestTechDis may be difficult to use, since the computational time needed to solve its LP relaxation is very large in most cases, especially for the large asymmetric instances of the second group. This may be an 
Table 2 Comparison in terms of optimality gap: first group (40 nodes-tra-trs)

\begin{tabular}{|c|c|c|c|c|}
\hline Instance & AggIPTime & Agg & DestDis & DestTechDis \\
\hline tra40-1-20-4-16.dat & 1.89 & 4.47 & 0.36 & 0.00 \\
\hline tra40-1-20-16-4.dat & 71.87 & 16.69 & 7.99 & 2.50 \\
\hline tra40-1-4-20-16.dat & 1.51 & 3.76 & 1.65 & 0.05 \\
\hline tra40-1-16-20-4.dat & 106.65 & 12.52 & 7.57 & 1.68 \\
\hline tra40-1-4-16-20.dat & 2.32 & 7.34 & 3.99 & 1.07 \\
\hline tra40-1-16-4-20.dat & 1.01 & 7.63 & 1.01 & 0.18 \\
\hline tra40-1-20-12-8.dat & 197.02 & 16.39 & 7.55 & 4.20 \\
\hline tra40-1-20-8-12.dat & 11.98 & 8.43 & 5.93 & 0.60 \\
\hline tra40-1-8-20-12.dat & 57.98 & 9.84 & 5.37 & 1.60 \\
\hline tra40-1-12-20-8.dat & 11.16 & 9.97 & 6.67 & 1.37 \\
\hline tra40-1-8-12-20.dat & 0.70 & 4.53 & 2.51 & 0.00 \\
\hline tra40-1-12-8-20.dat & 21.58 & 6.15 & 1.85 & 0.91 \\
\hline tra40-1-16-16-8.dat & 142.26 & 13.88 & 8.02 & 2.91 \\
\hline tra40-1-8-16-16.dat & 5.03 & 8.40 & 4.15 & 0.48 \\
\hline tra40-1-16-8-16.dat & 3.32 & 7.20 & 4.44 & 0.37 \\
\hline tra40-1-16-12-12.dat & 19.12 & 8.67 & 3.02 & 0.55 \\
\hline tra40-1-12-16-12.dat & 36.17 & 11.08 & 7.09 & 2.51 \\
\hline tra40-1-12-12-16.dat & 5.78 & 7.97 & 3.40 & 0.00 \\
\hline Avg tra40-1 & 38.74 & 9.16 & 4.59 & 1.17 \\
\hline $\operatorname{trs} 40-1-20-4-16 . d a t$ & 184.28 & 29.94 & 5.61 & 0.12 \\
\hline trs40-1-20-16-4.dat & 359.20 & 38.00 & 10.30 & 1.61 \\
\hline $\operatorname{trs} 40-1-4-20-16 . d a t$ & 1.59 & 21.26 & 1.95 & 0.00 \\
\hline trs40-1-16-20-4.dat & 130.64 & 30.35 & 5.36 & 0.63 \\
\hline $\operatorname{trs} 40-1-4-16-20 . d a t$ & 0.87 & 18.79 & 1.55 & 0.00 \\
\hline trs40-1-16-4-20.dat & 12.56 & 28.35 & 3.05 & 0.00 \\
\hline $\operatorname{trs} 40-1-20-12-8 . d a t$ & $1,930.65$ & 32.66 & 10.42 & 1.82 \\
\hline trs40-1-20-8-12.dat & 56.90 & 33.34 & 6.35 & 1.31 \\
\hline trs40-1-8-20-12.dat & 115.75 & 26.14 & 6.09 & 0.14 \\
\hline trs40-1-12-20-8.dat & 761.10 & 35.89 & 7.93 & 1.91 \\
\hline trs40-1-8-12-20.dat & 2.10 & 20.40 & 4.28 & 0.00 \\
\hline $\operatorname{trs} 40-1-12-8-20 . d a t$ & 65.60 & 22.66 & 3.58 & 0.00 \\
\hline trs40-1-16-16-8.dat & $2,618.03$ & 35.05 & 8.30 & 0.85 \\
\hline trs40-1-8-16-16.dat & 0.98 & 24.99 & 3.73 & 0.00 \\
\hline $\operatorname{trs} 40-1-16-8-16 . d a t$ & 15.64 & 28.70 & 5.22 & 0.71 \\
\hline trs40-1-16-12-12.dat & 167.81 & 29.89 & 8.90 & 1.65 \\
\hline trs40-1-12-16-12.dat & 241.72 & 29.05 & 6.50 & 0.70 \\
\hline $\operatorname{trs} 40-1-12-12-16 . d a t$ & 250.82 & 31.98 & 8.06 & 0.00 \\
\hline Avg trs $40-1$ & 384.24 & 28.75 & 5.95 & 0.64 \\
\hline
\end{tabular}

issue in real applications where the number of the nodes is usually large and where, in addition, the number of involved skills and technicians can be larger than 3 , which is the case addressed in the first pool of experiments. 
Table 3 Comparison in terms of optimality gap: second group

\begin{tabular}{|c|c|c|c|c|}
\hline Instance & AggIPTime & Agg & DestDis & DestTechDis \\
\hline A045-03f-d2-23-18-4.dat & $21,732.59$ & 19.68 & 8.45 & 3.25 \\
\hline A045-03f-d2-18-23-4.dat & $10,982.91$ & 18.40 & 6.79 & 2.01 \\
\hline A045-03f-d2-23-13-9.dat & $1,154.37$ & 17.75 & 6.18 & 0.00 \\
\hline A045-03f-d2-13-23-9.dat & $5,563.51$ & 17.54 & 5.90 & 2.75 \\
\hline A045-03f-d2-18-18-9.dat & $5,247.59$ & 18.82 & 7.36 & 3.40 \\
\hline A045-03f-d2-19-13-13.dat & $1,487.40$ & 13.43 & 4.35 & 0.10 \\
\hline Avg A045 & $7,694.73$ & 17.60 & 6.51 & 1.92 \\
\hline A048-03f-d2-24-19-5.dat & $21,730.29$ & 21.87 & 7.14 & 1.63 \\
\hline A048-03f-d2-19-24-5.dat & $18,952.51$ & 18.95 & 5.91 & 1.55 \\
\hline A048-03f-d2-24-15-9.dat & $21,743.53$ & 22.59 & 10.65 & 6.93 \\
\hline A048-03f-d2-15-24-9.dat & $2,464.10$ & 14.34 & 3.55 & 0.00 \\
\hline A048-03f-d2-19-19-10.dat & $16,649.30$ & 21.01 & 7.08 & 3.21 \\
\hline A048-03f-d2-20-14-14.dat & $21,648.84$ & 17.54 & 6.50 & 3.35 \\
\hline Avg A048 & $17,198.09$ & 19.38 & 6.81 & 2.78 \\
\hline A056-03f-d2-28-22-6.dat & $21,727.61$ & 31.10 & 13.59 & 10.18 \\
\hline A056-03f-d2-22-28-6.dat & $21,698.00$ & 21.92 & 5.45 & 1.64 \\
\hline A056-03f-d2-28-17-11.dat & $21,716.66$ & 29.23 & 15.50 & 6.65 \\
\hline A056-03f-d2-17-28-11.dat & $16,131.78$ & 16.40 & 5.52 & 1.93 \\
\hline A056-03f-d2-22-22-12.dat & $20,915.98$ & 20.92 & 6.19 & 2.28 \\
\hline A056-03f-d2-22-17-17.dat & $21,697.77$ & 20.25 & 7.68 & 2.74 \\
\hline Avg A056 & $20,647.97$ & 23.30 & 8.99 & 4.24 \\
\hline A065-03f-d2-33-26-6.dat & $43,200.00$ & 30.55 & 17.62 & 12.04 \\
\hline A065-03f-d2-26-33-6.dat & $43,200.00$ & 29.67 & 15.69 & 9.87 \\
\hline A065-03f-d2-33-19-13.dat & $43,200.00$ & 17.21 & 6.98 & 3.53 \\
\hline A065-03f-d2-19-33-13.dat & $43,200.00$ & 17.08 & 9.20 & 3.36 \\
\hline A065-03f-d2-26-26-13.dat & $43,200.00$ & 20.21 & 10.25 & 4.75 \\
\hline A065-03f-d2-25-20-20.dat & $43,200.00$ & 17.26 & 7.48 & 3.02 \\
\hline Avg A065 & $43,200.00$ & 22.00 & 11.20 & 6.10 \\
\hline A071-03f-d2-36-28-7.dat & $43,200.00$ & 24.59 & 13.60 & 7.70 \\
\hline A071-03f-d2-28-36-7.dat & $43,200.00$ & 16.37 & 3.93 & 0.44 \\
\hline A071-03f-d2-36-21-14.dat & $43,200.00$ & 20.22 & 12.36 & 7.10 \\
\hline A071-03f-d2-21-36-14.dat & $43,200.00$ & 20.28 & 9.94 & 3.71 \\
\hline A071-03f-d2-28-28-15.dat & $43,200.00$ & 21.44 & 9.83 & 4.32 \\
\hline A071-03f-d2-29-21-21.dat & $43,200.00$ & 15.60 & 9.02 & 4.66 \\
\hline Avg A071 & $43,200.00$ & 19.75 & 9.78 & 4.66 \\
\hline
\end{tabular}

In order to achieve a compromise between strength of the LP bound and computational effort required to compute it, in the successive phase of our computational analysis we have also evaluated the weaker models Agg and DestDis enhanced with the addition of the valid inequalities TC1 and TC2, presented in "Special cut constraints". This will be described in the next section. 
Table 4 Comparison in terms of average time: first group (40 nodes)

\begin{tabular}{lrlll}
\hline Instance & AggIP & Agg & DestDis & DestTechDis \\
\hline tc40-1 & $12,085.05$ & 0.24 & 598.25 & 721.08 \\
te40-1 & $12,165.09$ & 0.26 & 459.85 & 855.29 \\
tra40-1 & 38.74 & 0.37 & 206.61 & 168.02 \\
trs40-1 & 384.24 & 0.29 & 752.12 & 805.23 \\
\hline
\end{tabular}

Table 5 Comparison in terms of average time: second group

\begin{tabular}{lrlrr}
\hline Instance & \multicolumn{1}{c}{ AggIP } & Agg & DestDis & DestTechDis \\
\hline A045 & $7,694.73$ & 0.42 & 306.21 & 533.41 \\
A048 & $17,198.09$ & 0.48 & 598.61 & $1,000.25$ \\
A056 & $20,647.97$ & 1.40 & $1,314.40$ & $3,031.66$ \\
A065 & $43,200.00$ & 3.72 & $3,039.72$ & $9,266.48$ \\
A071 & $43,200.00$ & 5.71 & $3,902.66$ & $15,537.47$ \\
\hline
\end{tabular}

Assessing the impact of valid inequalities

We have conducted some tests to evaluate the strength of the valid inequalities TC1 and TC2 when added to models Agg and DestDis on the two groups of A-SKVRP instances described in "A-SKVRP instances: scenario with 3 skills". In Tables 6, 7 and 8 a comparison of the models StrongAgg, StrongDestDis (i.e. Agg and DestDis equipped with TC1 and TC2) and DestTechDis is given for the 40 nodes instances of the first group in terms of percentage gap of the LP bound with respect to the optimum value (which has been computed via model Agg, as remarked above) and of average time required to solve the continuous relaxation. Analogous results are provided in Tables 9 and 10 for the asymmetric instances of the second group.

The results show that TC1 and TC2 are indeed very effective. The introduction of these inequalities in model Agg leads to reductions of the average percentage gap from 29.64 to 13.33 for the instances of type $t c$, and from 26.03 to 9.29 for the instances of type te. Drastic gap improvements are achieved also for the other sets of instances, including the asymmetric instances of the second group. Substantial reductions are obtained also by StrongDestDis. These results are summarized in Tables 11 and 12 .

It is worth observing that the intermediate model is in most cases much faster after the addition of cuts TC1 and TC2, i.e. StrongDestDis is faster than DestDis, as the average times in Tables 8 and 10 show (this occurs for the instances of type $t c$, te and tra, and even more for all the instances in the second group).

Also note that model DestDis, when enhanced with TC1 and TC2, is able to find a solution of optimal value in 9 over 72 cases in the first group of instances; on the contrary, model DestDis without any cuts is unable to find the optimum within the same time limit.

As already observed, the instances of type tra and trs of the first group of instances appear to be easy to solve to optimality in most cases (see Table 7). 
Table 6 TC1 and TC2-comparison in terms of optimality gap: first group (40 nodes-tc-te)

\begin{tabular}{|c|c|c|c|c|}
\hline Instance & AggIPTime & StrongAgg & StrongDestDis & DestTechDis \\
\hline tc $40-1-20-4-16 . d a t$ & $21,741.87$ & 12.32 & 4.04 & 1.92 \\
\hline tc40-1-20-16-4.dat & $21,699.26$ & 21.61 & 7.78 & 3.23 \\
\hline tc $40-1-4-20-16 . d a t$ & $2,865.86$ & 11.05 & 1.85 & 0.30 \\
\hline tc40-1-16-20-4.dat & $21,691.04$ & 21.88 & 7.42 & 4.41 \\
\hline tc40-1-4-16-20.dat & 742.10 & 7.14 & 0.85 & 0.00 \\
\hline tc40-1-16-4-20.dat & $4,907.78$ & 9.15 & 2.75 & 0.73 \\
\hline tc $40-1-20-12-8$.dat & $21,718.93$ & 18.15 & 6.71 & 3.52 \\
\hline tc40-1-20-8-12.dat & $19,093.48$ & 15.04 & 1.09 & 0.18 \\
\hline tc40-1-8-20-12.dat & $21,653.45$ & 13.02 & 2.03 & 0.59 \\
\hline tc40-1-12-20-8.dat & $21,694.86$ & 17.61 & 4.21 & 1.79 \\
\hline tc40-1-8-12-20.dat & 175.17 & 6.93 & 0.12 & 0.00 \\
\hline tc40-1-12-8-20.dat & $4,476.51$ & 12.42 & 2.30 & 1.22 \\
\hline tc40-1-16-16-8.dat & $21,695.64$ & 20.51 & 6.45 & 4.36 \\
\hline tc40-1-8-16-16.dat & 985.21 & 6.60 & 0.00 & 0.00 \\
\hline tc40-1-16-8-16.dat & $3,992.99$ & 10.86 & 1.92 & 0.82 \\
\hline tc $40-1-16-12-12 . d a t$ & $3,469.94$ & 12.08 & 1.21 & 0.13 \\
\hline tc $40-1-12-16-12 . d a t$ & $21,742.12$ & 15.60 & 2.96 & 0.49 \\
\hline tc $40-1-12-12-16 . d a t$ & $3,184.70$ & 7.92 & 0.00 & 0.00 \\
\hline Avg tc40-1 & $12,085.05$ & 13.33 & 2.98 & 1.32 \\
\hline te $40-1-20-4-16 . d a t$ & $1,192.86$ & 5.59 & 2.39 & 0.38 \\
\hline te $40-1-20-16-4 . d a t$ & $21,709.62$ & 21.66 & 11.62 & 8.85 \\
\hline te $40-1-4-20-16 . d a t$ & 20.46 & 2.32 & 1.45 & 0.00 \\
\hline te $40-1-16-20-4 . d a t$ & $21,709.06$ & 21.19 & 11.17 & 8.78 \\
\hline te $40-1-4-16-20 . d a t$ & 7.31 & 0.90 & 0.21 & 0.00 \\
\hline te $40-1-16-4-20 . d a t$ & $2,611.68$ & 4.82 & 1.39 & 0.28 \\
\hline te $40-1-20-12-8 . d a t$ & $21,701.03$ & 15.89 & 5.86 & 4.33 \\
\hline te $40-1-20-8-12 . d a t$ & $21,721.15$ & 9.49 & 3.18 & 0.00 \\
\hline te $40-1-8-20-12 . d a t$ & $21,704.39$ & 17.99 & 11.02 & 9.44 \\
\hline te $40-1-12-20-8 . d a t$ & $21,697.92$ & 14.64 & 5.85 & 3.79 \\
\hline te $40-1-8-12-20 . d a t$ & 181.85 & 1.33 & 0.83 & 0.00 \\
\hline te $40-1-12-8$-20.dat & 154.84 & 2.02 & 1.57 & 0.00 \\
\hline te $40-1-16-16-8 . d a t$ & $21,739.62$ & 12.56 & 7.02 & 2.66 \\
\hline te $40-1-8-16-16 . d a t$ & $2,729.12$ & 3.85 & 1.96 & 0.42 \\
\hline te $40-1-16-8$-16.dat & $21,757.85$ & 8.33 & 4.36 & 2.09 \\
\hline te40-1-16-12-12.dat & $6,681.37$ & 6.66 & 0.26 & 0.00 \\
\hline te $40-1-12-16-12 . d a t$ & $21,731.71$ & 11.57 & 5.94 & 2.49 \\
\hline te40-1-12-12-16.dat & $9,919.81$ & 6.44 & 3.99 & 0.30 \\
\hline Avg te $40-1$ & $12,165.09$ & 9.29 & 4.45 & 2.43 \\
\hline
\end{tabular}

Therefore, the instances of type tra and trs have not been considered anymore in the rest of the experimentation. On the other hand, the instances of type $t c$ and te appear to be more difficult to solve. The same observation holds for the instances of the 
Table 7 TC1 and TC2—comparison in terms of optimality gap: first group (40 nodes-tra-trs)

\begin{tabular}{|c|c|c|c|c|}
\hline Instance & AggIPTime & StrongAgg & StrongDestDis & DestTechDis \\
\hline $\operatorname{tra} 40-1-20-4-16 . d a t$ & 1.89 & 0.19 & 0.00 & 0.00 \\
\hline tra40-1-20-16-4.dat & 71.87 & 2.54 & 2.54 & 2.50 \\
\hline tra40-1-4-20-16.dat & 1.51 & 0.16 & 0.11 & 0.05 \\
\hline tra40-1-16-20-4.dat & 106.65 & 1.71 & 1.71 & 1.68 \\
\hline tra40-1-4-16-20.dat & 2.32 & 1.22 & 1.07 & 1.07 \\
\hline tra40-1-16-4-20.dat & 1.01 & 0.70 & 0.22 & 0.18 \\
\hline tra40-1-20-12-8.dat & 197.02 & 4.63 & 4.49 & 4.20 \\
\hline tra40-1-20-8-12.dat & 11.98 & 1.01 & 0.75 & 0.60 \\
\hline tra40-1-8-20-12.dat & 57.98 & 2.00 & 2.00 & 1.60 \\
\hline tra40-1-12-20-8.dat & 11.16 & 1.37 & 1.37 & 1.37 \\
\hline tra40-1-8-12-20.dat & 0.70 & 0.00 & 0.00 & 0.00 \\
\hline tra40-1-12-8-20.dat & 21.58 & 1.60 & 1.17 & 0.91 \\
\hline tra40-1-16-16-8.dat & 142.26 & 3.75 & 3.39 & 2.91 \\
\hline tra40-1-8-16-16.dat & 5.03 & 0.86 & 0.48 & 0.48 \\
\hline tra40-1-16-8-16.dat & 3.32 & 0.74 & 0.38 & 0.37 \\
\hline tra40-1-16-12-12.dat & 19.12 & 1.40 & 0.80 & 0.55 \\
\hline tra40-1-12-16-12.dat & 36.17 & 3.73 & 3.73 & 2.51 \\
\hline tra40-1-12-12-16.dat & 5.78 & 0.34 & 0.13 & 0.00 \\
\hline Avg tra40-1 & 38.74 & 1.55 & 1.35 & 1.17 \\
\hline $\operatorname{trs} 40-1-20-4-16 . d a t$ & 184.28 & 1.95 & 1.33 & 0.12 \\
\hline trs40-1-20-16-4.dat & 359.20 & 3.04 & 2.73 & 1.61 \\
\hline $\operatorname{trs} 40-1-4-20-16 . d a t$ & 1.59 & 0.14 & 0.00 & 0.00 \\
\hline $\operatorname{trs} 40-1-16-20-4 . d a t$ & 130.64 & 3.06 & 1.53 & 0.63 \\
\hline $\operatorname{trs} 40-1-4-16-20 . d a t$ & 0.87 & 0.00 & 0.00 & 0.00 \\
\hline trs40-1-16-4-20.dat & 12.56 & 1.68 & 1.15 & 0.00 \\
\hline trs40-1-20-12-8.dat & $1,930.65$ & 4.02 & 3.18 & 1.82 \\
\hline trs40-1-20-8-12.dat & 56.90 & 2.31 & 1.84 & 1.31 \\
\hline $\operatorname{trs} 40-1-8-20-12 . d a t$ & 115.75 & 0.62 & 0.32 & 0.14 \\
\hline $\operatorname{trs} 40-1-12-20-8 . d a t$ & 761.10 & 3.90 & 3.40 & 1.91 \\
\hline trs40-1-8-12-20.dat & 2.10 & 0.01 & 0.00 & 0.00 \\
\hline $\operatorname{trs} 40-1-12-8-20 . d a t$ & 65.60 & 1.04 & 0.00 & 0.00 \\
\hline $\operatorname{trs} 40-1-16-16-8 . d a t$ & $2,618.03$ & 3.44 & 1.93 & 0.85 \\
\hline $\operatorname{trs} 40-1-8-16-16 . d a t$ & 0.98 & 0.00 & 0.00 & 0.00 \\
\hline trs40-1-16-8-16.dat & 15.64 & 2.11 & 1.83 & 0.71 \\
\hline trs40-1-16-12-12.dat & 167.81 & 3.98 & 3.72 & 1.65 \\
\hline trs40-1-12-16-12.dat & 241.72 & 2.78 & 2.57 & 0.70 \\
\hline $\operatorname{trs} 40-1-12-12-16 . d a t$ & 250.82 & 4.22 & 2.42 & 0.00 \\
\hline Avg trs $40-1$ & 384.24 & 2.13 & 1.55 & 0.64 \\
\hline
\end{tabular}

second group, of larger size. On these more difficult instances, the intermediate model StrongDestDis behaves much better than StrongAgg in terms of percentage gaps. Furthermore, after the cut addition the gaps of the intermediate model are 
Table 8 TC1 and TC2 - comparison in terms of average time: first group (40 nodes)

\begin{tabular}{lrlcl}
\hline Instance & AggIP & StrongAgg & StrongDestDis & DestTechDis \\
\hline tc40-1 & $12,085.05$ & 0.77 & 317.83 & 721.08 \\
te40-1 & $12,165.09$ & 0.83 & 263.03 & 855.29 \\
tra40-1 & 38.74 & 0.69 & 82.17 & 168.02 \\
trs40-1 & 384.24 & 1.17 & 218.30 & 805.23 \\
\hline
\end{tabular}

quite small (indeed, they are close to the ones produced by the strongest model DestTechDis, see Tables 6,9), and they are often still computed in a reasonable computational time (see Tables 8,10 ). These facts have motivated us to spend more time in enhancing the intermediate model since, among the proposed models, it appears to be a good compromise between efficacy and efficiency. This will be the subject of the next section, devoted to the further enhancement of model StrongDestDis via the two-cycle inequalities presented in "Two-cycle inequalities and related cutting plane approach".

Assessing the impact of the cutting plane approach

\section{Scenario with 40 nodes and 3 skills}

We firstly tested the cutting plane approach on the $t c$ instances with 40 nodes and 3 skills, described in "A-SKVRP instances: scenario with 3 skills". As observed before, among the A-SKVRP instances with 40 nodes, these $t c$ instances proved to be difficult to solve with the three models presented in "ILP models for the Asymmetric Skill VRP". Thus these instances define a good initial set for testing the strength of the two-cycle inequalities. In Table 13, we report the optimality gap related to the first iteration of the cutting plane approach, as well as the one obtained after four iterations (experimentally we observed that, for the considered instances, the improvement is negligible after the fourth iteration). Also the optimality gap obtained by the models StrongDestDis and DestTechDis is given. We observe that the addition of the two-cycle inequalities improves on the gap returned by model StrongDestDis in all cases. In some cases the improvement is remarkable allowing us to conclude that these inequalities qualify as an interesting tool to approximate the very good LP bound provided by DestTechDis. The computational times required by the cutting plane approach on the considered instances were, however, not competitive with the ones required by DestTechDis, essentially due to the number of nodes, which is high but still tractable, and to the limited number of skills and technicians characterizing the considered instances. In fact, model DestTechDis is solvable in less than $1,000 \mathrm{~s}$ (see Table 8).

As already observed, in application contexts such as Field Service and Home Care, the number of nodes may be larger than 40 and the number of involved skills may be larger than 3. Increasing the number of the nodes becomes critical for the strongest model DestTechDis, as shown by the results in the previous sections concerning the large instances of the second group. Also, increasing the number of 
Table 9 TC1 and TC2 - comparison in terms of optimality gap: second group

\begin{tabular}{|c|c|c|c|c|}
\hline Instance & AggIPTime & StrongAgg & StrongDestDis & DestTechDis \\
\hline A045-03f-d2-23-18-4.dat & $21,732.59$ & 11.65 & 5.27 & 3.25 \\
\hline A045-03f-d2-18-23-4.dat & $10,982.91$ & 9.15 & 3.75 & 2.01 \\
\hline A045-03f-d2-23-13-9.dat & $1,154.37$ & 4.95 & 2.99 & 0.00 \\
\hline A045-03f-d2-13-23-9.dat & $5,563.51$ & 10.03 & 3.55 & 2.75 \\
\hline A045-03f-d2-18-18-9.dat & $5,247.59$ & 6.86 & 4.26 & 3.40 \\
\hline A045-03f-d2-19-13-13.dat & $1,487.40$ & 6.31 & 1.46 & 0.10 \\
\hline Avg A045 & $7,694.73$ & 8.16 & 3.55 & 1.92 \\
\hline A048-03f-d2-24-19-5.dat & $21,730.29$ & 11.27 & 4.22 & 1.63 \\
\hline A048-03f-d2-19-24-5.dat & $18,952.51$ & 6.59 & 3.24 & 1.55 \\
\hline A048-03f-d2-24-15-9.dat & $21,743.53$ & 12.05 & 7.18 & 6.93 \\
\hline A048-03f-d2-15-24-9.dat & $2,464.10$ & 3.34 & 0.04 & 0.00 \\
\hline A048-03f-d2-19-19-10.dat & $16,649.30$ & 9.34 & 4.70 & 3.21 \\
\hline A048-03f-d2-20-14-14.dat & $21,648.84$ & 6.85 & 4.07 & 3.35 \\
\hline Avg A048 & $17,198.09$ & 8.24 & 3.91 & 2.78 \\
\hline A056-03f-d2-28-22-6.dat & $21,727.61$ & 22.05 & 11.17 & 10.18 \\
\hline A056-03f-d2-22-28-6.dat & $21,698.00$ & 14.99 & 3.06 & 1.64 \\
\hline A056-03f-d2-28-17-11.dat & $21,716.66$ & 21.10 & 12.10 & 6.65 \\
\hline A056-03f-d2-17-28-11.dat & $16,131.78$ & 8.09 & 3.58 & 1.93 \\
\hline A056-03f-d2-22-22-12.dat & $20,915.98$ & 10.51 & 3.70 & 2.28 \\
\hline A056-03f-d2-22-17-17.dat & $21,697.77$ & 11.65 & 4.88 & 2.74 \\
\hline Avg A056 & $20,647.97$ & 14.73 & 6.41 & 4.24 \\
\hline A065-03f-d2-33-26-6.dat & $43,200.00$ & 23.22 & 14.52 & 12.04 \\
\hline A065-03f-d2-26-33-6.dat & $43,200.00$ & 20.79 & 12.59 & 9.87 \\
\hline A065-03f-d2-33-19-13.dat & $43,200.00$ & 10.26 & 5.61 & 3.53 \\
\hline A065-03f-d2-19-33-13.dat & $43,200.00$ & 8.40 & 4.86 & 3.36 \\
\hline A065-03f-d2-26-26-13.dat & $43,200.00$ & 12.66 & 6.94 & 4.75 \\
\hline A065-03f-d2-25-20-20.dat & $43,200.00$ & 7.35 & 4.51 & 3.02 \\
\hline Avg A065 & $43,200.00$ & 13.78 & 8.17 & 6.10 \\
\hline A071-03f-d2-36-28-7.dat & $43,200.00$ & 17.32 & 11.06 & 7.70 \\
\hline A071-03f-d2-28-36-7.dat & $43,200.00$ & 6.80 & 1.57 & 0.44 \\
\hline A071-03f-d2-36-21-14.dat & $43,200.00$ & 12.97 & 9.84 & 7.10 \\
\hline A071-03f-d2-21-36-14.dat & $43,200.00$ & 12.10 & 7.22 & 3.71 \\
\hline A071-03f-d2-28-28-15.dat & $43,200.00$ & 11.00 & 7.96 & 4.32 \\
\hline A071-03f-d2-29-21-21.dat & $43,200.00$ & 10.30 & 6.36 & 4.66 \\
\hline Avg A071 & $43,200.00$ & 11.75 & 7.33 & 4.66 \\
\hline
\end{tabular}

the skills becomes critical for DestTechDis, whose size increases with the number of skills and technicians. Therefore, in the last phase of the computational experience we have investigated the effectiveness of the cutting plane approach as the number of nodes and skills increases. 
Table 10 TC1 and TC2 - comparison in terms of average time: second group

\begin{tabular}{lllcr}
\hline Instance & AggIP & StrongAgg & StrongDestDis & DestTechDis \\
\hline A045 & $7,694.73$ & 1.03 & 218.58 & 533.41 \\
A048 & $17,198.09$ & 1.65 & 303.81 & $1,000.25$ \\
A056 & $20,647.97$ & 2.81 & 539.09 & $3,031.66$ \\
A065 & $43,200.00$ & 5.87 & $1,929.58$ & $9,266.48$ \\
A071 & $43,200.00$ & 7.22 & $2,454.96$ & $15,537.47$ \\
\hline
\end{tabular}

Table 11 TC1 and TC2 - comparison in terms of average gap: first group (40 nodes)

\begin{tabular}{lrllll}
\hline Instance & Agg & StrongAgg & DestDis & StrongDestDis & DestTechDis \\
\hline tc40-1 & 29.64 & 13.33 & 5.62 & 2.98 & 1.32 \\
te40-1 & 26.03 & 9.29 & 8.89 & 4.45 & 2.43 \\
tra40-1 & 9.16 & 1.55 & 4.59 & 1.35 & 1.17 \\
trs40-1 & 28.75 & 2.13 & 5.95 & 1.55 & 0.64 \\
\hline
\end{tabular}

Table 12 TC1 and TC2 - comparison in terms of average gap: second group

\begin{tabular}{lllcll}
\hline Instance & Agg & StrongAgg & DestDis & StrongDestDis & DestTechDis \\
\hline A045 & 17.60 & 8.16 & 6.51 & 3.55 & 1.92 \\
A048 & 19.38 & 8.24 & 6.81 & 3.91 & 2.78 \\
A056 & 23.30 & 14.73 & 8.99 & 6.41 & 4.24 \\
A065 & 22.00 & 13.78 & 11.20 & 8.17 & 6.10 \\
A071 & 19.75 & 11.75 & 9.78 & 7.33 & 4.66 \\
\hline
\end{tabular}

\section{Trend for increasing number of nodes}

We tested the effectiveness of the cutting plane approach as the number of the nodes increases by addressing the largest instances of the second group, i.e. the ones with 56, 65 and 71 nodes. A comparison of the models StrongDestDis, StrongDestDis enhanced with the cutting plane approach and DestTechDis is given in terms of percentage optimality gap in Table 14, and in terms of average time required to solve the continuous relaxation in Table 15 . Specifically, we provide the optimality gap and the computational time related to the first iteration of the cutting plane approach (StrongDestDis+CP).

As observed, the time of DestTechDis grows very fast, requiring more than $4 \mathrm{~h}$ on average on the largest instances with 71 nodes (see Table 15). Therefore, the use of model DestTechDis is not suitable for instances with a large number of nodes. On the other hand, the cutting plane approach improves on the gap returned by model StrongDestDis (except only 2 cases) within a time which is about one half the time required by the strongest model. Concerning the optimality gap, StrongDestDis 
Table 13 Optimality gap via cutting plane (40 nodes-tc-3 skills)

\begin{tabular}{lllll}
\hline Instance & StrongDestDis & 1 iteration & 4 iterations & DestTechDis \\
\hline tc40-1-20-4-16.dat & 4.04 & 3.28 & 2.55 & 1.92 \\
tc40-1-20-16-4.dat & 7.78 & 6.63 & 5.46 & 3.23 \\
tc40-1-4-20-16.dat & 1.85 & 1.36 & 0.54 & 0.30 \\
tc40-1-16-20-4.dat & 7.42 & 6.71 & 6.27 & 4.41 \\
tc40-1-4-16-20.dat & 0.85 & 0.00 & 0.00 & 0.00 \\
tc40-1-16-4-20.dat & 2.75 & 1.99 & 1.77 & 0.73 \\
tc40-1-20-12-8.dat & 6.71 & 6.08 & 6.08 & 3.52 \\
tc40-1-20-8-12.dat & 1.09 & 0.45 & 0.44 & 0.18 \\
tc40-1-8-20-12.dat & 2.03 & 1.85 & 1.85 & 0.59 \\
tc40-1-12-20-8.dat & 4.21 & 3.58 & 2.45 & 1.79 \\
tc40-1-8-12-20.dat & 0.12 & 0.00 & 0.00 & 0.00 \\
tc40-1-12-8-20.dat & 2.30 & 2.30 & 2.17 & 1.22 \\
tc40-1-16-16-8.dat & 6.45 & 5.83 & 5.73 & 4.36 \\
tc40-1-8-16-16.dat & 0.00 & 0.00 & 0.00 & 0.00 \\
tc40-1-16-8-16.dat & 1.92 & 1.01 & 1.01 & 0.82 \\
tc40-1-16-12-12.dat & 1.21 & 0.76 & 0.19 & 0.13 \\
tc40-1-12-16-12.dat & 2.96 & 2.33 & 1.28 & 0.49 \\
tc40-1-12-12-16.dat & 0.00 & 0.00 & 0.00 & $\mathbf{2 . 1 0}$ \\
Avg tc40-1 & $\mathbf{2 . 9 8}$ & $\mathbf{2 . 4 5}$ & \\
\hline Avera & & & \\
\hline
\end{tabular}

Average values are reported in bold

enhanced with the cutting plane provides gaps whose difference, with respect to the ones found by DestTechDis, is less than $2 \%$ in most cases, while the average improvement with respect to the gap provided by StrongDestDis is at least $0.38 \%$ (see Table 14).

\section{Trend for increasing number of skills}

We generated two additional sets of A-SKVRP instances, with, respectively, 6 and 9 skills and technicians, starting from the $t c$ instance number one with 40 nodes. In the case of 6 skills, we generated 8 A-SKVRP instances according to the following patterns used to assign a skill to the nodes:

25-15-15-15-15-15 (for a total of 6 permutations)

50-10-10-10-10-10 (only the 2 extreme permutations 50-10-10-10-10-10 and $10-10-10-10-10-50)$

where the numbers indicate the percentage of nodes requiring a specific skill. The last two extreme permutations have been generated in order to evaluate the impact of the pattern assignment on the difficulty of A-SKVRP instances (the intuition is that pattern 50-10-10-10-10-10 should be more difficult than 10-10-10-10-10-50, since there are more choices in the assignment of technicians to nodes requiring the 
Table 14 Comparison in terms of optimality gap: 56, 65 and 71 nodes

\begin{tabular}{|c|c|c|c|}
\hline Instance & StrongDestDis & StrongDestDis +CP & DestTechDis \\
\hline A056-03f-d2-28-22-6.dat & 11.17 & 10.86 & 10.18 \\
\hline A056-03f-d2-22-28-6.dat & 3.06 & 2.99 & 1.64 \\
\hline A056-03f-d2-28-17-11.dat & 12.10 & 10.62 & 6.65 \\
\hline A056-03f-d2-17-28-11.dat & 3.58 & 3.30 & 1.93 \\
\hline A056-03f-d2-22-22-12.dat & 3.70 & 3.60 & 2.28 \\
\hline A056-03f-d2-22-17-17.dat & 4.88 & 4.55 & 2.74 \\
\hline Avg A056 & 6.41 & 5.99 & 4.24 \\
\hline A065-03f-d2-33-26-6.dat & 14.52 & 13.75 & 12.04 \\
\hline A065-03f-d2-26-33-6.dat & 12.59 & 11.75 & 9.87 \\
\hline A065-03f-d2-33-19-13.dat & 5.61 & 5.28 & 3.53 \\
\hline A065-03f-d2-19-33-13.dat & 4.86 & 4.68 & 3.36 \\
\hline A065-03f-d2-26-26-13.dat & 6.94 & 6.94 & 4.75 \\
\hline A065-03f-d2-25-20-20.dat & 4.51 & 4.35 & 3.02 \\
\hline Avg A065 & 8.17 & 7.79 & 6.10 \\
\hline A071-03f-d2-36-28-7.dat & 11.06 & 10.67 & 7.70 \\
\hline A071-03f-d2-28-36-7.dat & 1.57 & 1.57 & 0.44 \\
\hline A071-03f-d2-36-21-14.dat & 9.84 & 9.58 & 7.10 \\
\hline A071-03f-d2-21-36-14.dat & 7.22 & 6.01 & 3.71 \\
\hline A071-03f-d2-28-28-15.dat & 7.96 & 7.53 & 4.32 \\
\hline A071-03f-d2-29-21-21.dat & 6.36 & 6.34 & 4.66 \\
\hline Avg A071 & 7.33 & 6.95 & 4.66 \\
\hline
\end{tabular}

Average values are reported in bold

Table 15 Comparison in terms of average time: 56,65 and 71 nodes

\begin{tabular}{lccc}
\hline Instance & StrongDestDis & StrongDestDis+CP & DestTechDis \\
\hline A056 & 539.09 & $1,669.61$ & $3,031.66$ \\
A065 & $1,929.58$ & $4,371.94$ & $9,266.48$ \\
A071 & $2,454.96$ & $6,082.60$ & $15,537.47$ \\
\hline
\end{tabular}

lowest ability). In the case of nine skills, we used the pattern 20-10-10-10-10-10-1010-10, for a total of 9 permutations.

The formula used to generate the costs is the one described in "A-SKVRP instances: scenario with 3 skills".

Also in this case a comparison of the models StrongDestDis, StrongDestDis enhanced via the cutting plane approach and DestTechDis is given in terms of percentage gap of the LP bound with respect to the best integer solution computed via model Agg with a time limit of $6 \mathrm{~h}$ in Tables 16 and 17, and in terms of average time required to solve the continuous relaxation in Table 18. Specifically, we provide the optimality gap and the computational time related to the first iteration of the cutting plane approach (StrongDestDis+CP). 
Table 16 Comparison in terms of optimality gap: 6 skills

\begin{tabular}{llll}
\hline Instance & StrongDestDis & StrongDestDis+CP & DestTechDis \\
\hline tc40-1-10-6-6-6-6-6.dat & 7.55 & 7.10 & 5.47 \\
tc40-1-6-10-6-6-6-6.dat & 8.12 & 6.99 & 4.99 \\
tc40-1-6-6-10-6-6-6.dat & 2.73 & 1.54 & 1.31 \\
tc40-1-6-6-6-10-6-6.dat & 1.05 & 0.69 & 0.00 \\
tc40-1-6-6-6-6-10-6.dat & 7.87 & 6.45 & 5.17 \\
tc40-1-6-6-6-6-6-10.dat & 0.06 & 0.00 & 0.00 \\
tc40-1-20-4-4-4-4-4.dat & 4.56 & 3.72 & 1.15 \\
tc40-1-4-4-4-4-4-20.dat & 0.00 & 0.00 & 0.00 \\
Avg tc40-1 & $\mathbf{3 . 9 9}$ & $\mathbf{3 . 3 1}$ & $\mathbf{2 . 2 6}$ \\
Avg tc40-1 Critical & $\mathbf{6 . 5 7}$ & $\mathbf{5 . 5 2}$ & $\mathbf{4 . 2 3}$ \\
\hline
\end{tabular}

Average values are reported in bold

Table 17 Comparison in terms of optimality gap: 9 skills

\begin{tabular}{lccc}
\hline Instance & StrongDestDis & StrongDestDis+CP & DestTechDis \\
\hline tc40-1-8-4-4-4-4-4-4-4-4.dat & 8.22 & 7.81 & 6.26 \\
tc40-1-4-8-4-4-4-4-4-4-4.dat & 9.30 & 8.58 & 6.42 \\
tc40-1-4-4-8-4-4-4-4-4-4.dat & 2.01 & 1.74 & 0.63 \\
tc40-1-4-4-4-8-4-4-4-4-4.dat & 11.74 & 11.54 & 10.07 \\
tc40-1-4-4-4-4-8-4-4-4-4.dat & 7.49 & 6.64 & 3.14 \\
tc40-1-4-4-4-4-4-8-4-4-4.dat & 2.93 & 2.16 & 0.80 \\
tc40-1-4-4-4-4-4-4-8-4-4.dat & 2.76 & 2.36 & 1.71 \\
tc40-1-4-4-4-4-4-4-4-8-4.dat & 7.77 & 7.48 & 6.92 \\
tc40-1-4-4-4-4-4-4-4-4-8.dat & 1.49 & 1.22 & 0.04 \\
Avg tc40-1 & $\mathbf{5 . 9 7}$ & $\mathbf{5 . 5 0}$ & $\mathbf{4 . 0 0}$ \\
Avg tc40-1critical & $\mathbf{5 . 6 9}$ & $\mathbf{5 . 2 2}$ & $\mathbf{3 . 7 2}$ \\
\hline
\end{tabular}

Average values are reported in bold

Table 18 Comparison in terms of average time: 6 and 9 skills

\begin{tabular}{llll}
\hline 3Instance & StrongDestDis & StrongDestDis+CP & DestTechDis \\
\hline tc40-1: 6 skills & 469.17 & $2,006.83$ & $1,880.79$ \\
tc40-1: 6 skills critical & 545.98 & $1,516.62$ & $2,230.97$ \\
tc40-1: 9 skills & 620.87 & $2,309.82$ & $4,691.05$ \\
tc40-1: 9 skills critical & 638.21 & $1,750.94$ & $4,817.53$ \\
\hline
\end{tabular}

Tables 16, 17 and 18 also report the average statistics for the most critical instances with 6 and 9 skills, i.e. the ones for which the strongest model DestTechDis required more than 1,000 s to solve its LP relaxation (called Critical in these tables). Concerning the computational times it is possible to observe that model StrongDestDis is quite stable 
when the number of the skills increases, whereas the time required by DestTechDis grows very fast; the average time for the case of 6 skills is more than twice the average time for three skills, and the same trend can be observed going from 6 to 9 skills (see Tables 8, 18). This confirms that the use of model DestTechDis is not suitable for instances with a high number of skills and technicians. On the other hand, the cutting plane approach associated with the model StrongDestDis appears to be a good choice, especially for the critical instances in the two datasets. Concerning the optimality gap, StrongDestDis enhanced with the cutting plane provides gaps whose difference, with respect to the ones found by DestTechDis, is on average below $1.55 \%$ (see Tables 16, 17).

The intermediate model enhanced with the cut addition and strengthened by means of the cutting plane approach appears to be able to provide a lower bound of very good quality also on large A-SKVRP instances.

\section{Conclusions}

The problem of defining a set of tours, each one operated by a skilled technician, so as to fulfill service requirements asking for particular skills, has been defined and studied. Three models, characterized by an increasing level of variables disaggregation, have been formulated and tested on a large suite of randomly generated instances. The basic model is an adaptation of the single-commodity flow model proposed by Gavish and Graves (1979). The second model is an enhancement of the multicommodity version of the first model, while the third one is a "skilled-based" disaggregation of the second model. Computational results support a series of interesting observations. First, the third model produces very tight LP bounds. Second, the computational times required to solve the models increase with the complexity of the model, by indicating that the model producing the best LP bounds is difficult to use when attempting to solve instances with a large number of nodes and more than three levels of skill. Third, valid inequalities that are implied by the model with the strongest LP relaxation can be added to the weaker models leading to substantial improvements on the lower bounds provided by these models. In particular, the intermediate model, equipped with the proposed set of valid inequalities, appears to represent a good trade-off between quality of the LP bound and computational burden. Additionally, the behavior of the intermediate model can be improved further by a cutting plane approach up to the extent that it can be used successfully to address large scale A-SKVRP problems arising in challenging real application contexts. A study of the proposed models and valid inequalities in Home Care settings is ongoing.

Acknowledgments We are very grateful to Prof. Roberto Baldacci for the stimulating discussion on the relationships between the A-SKVRP and VRP problems from the literature. We also thank the two anonymous referees, the Associate Editor and the Guest Editors for the interesting comments and suggestions which allowed us to greatly improve the first version of the paper.

\section{References}

Agnihothri SR, Mishra AK (2004) Cross-training decisions in field services with three job types and server-job mismatch. Decis Sci 35(2):239-257 
Ahuja RK, Magnanti TL, Orlin JB (1993) Network flows: theory, algorithms, and applications. Prentice Hall, Englewood

Baldacci R, Bartolini E, Mingozzi A, Roberti R (2010a) An exact solution framework for a broad class of vehicle routing problems. Comput Manag Sci 7:229-268

Baldacci R, Bartolini E, Mingozzi A, Valletta A (2010b) An exact algorithm for the period routing problem. Oper Res 59:228-241

Baldacci R, Toth P, Vigo D (2010c) Exact algorithms for routing problems under vehicle capacity constraints. Ann Oper Res 175:213-245

Borsani V, Matta A, Beschi G, Sommaruga F (2006) A Home care scheduling model for human resources. In: IEEE international conference on service systems and service management, pp 449-454

Cappanera P, Gouveia L, Scutellà MG (2011) The skill vehicle routing problem. In: Pahl J, Reiners T, Voss S (eds) Network optimization. 5th international conference, INOC 2011, Hamburg, June 2011. Lecture notes in computer science, LNCS, Springer, Berlin, vol 6701, pp 354-364

Cordeau JF, Laporte G (2001) A Tabu Search algorithm for the site dependent vehicle routing problem with time windows. INFOR 39(3):321-354

Fischetti M, Toth P, Vigo D (1994) A branch-and-bound algorithm for the capacitated vehicle routing problem on directed graphs. Oper Res 42(5):846-859

Gavish B, Graves S (1979) The traveling salesman problem and related problems. Working Paper, Graduate School of Management, University of Rochester, New York

Godinho MT, Gouveia L, Magnanti T, Pesneau P, Pires J (2009) Inequalities implied by a time-dependent model for the unit demand vehicle routing problem. Working paper do CIO 10/2009

Gouveia L (1995) A $2 n$-constraint formulation for the capacitated minimal spanning tree problem. Oper Res 43:130-141

Gouveia L, Simonetti L, Uchoa E (2011) Hop-constrained and diameter-constrained minimum spanning tree problems as Steiner tree problems over layered graphs. Math Progr 128:123-148

Letchford A, Salazar-Gonzalez JJ (2006) Projection results for vehicle routing. Math Progr 105:251-274

Nag B, Golden BL, Assad AA (1988) Vehicle routing with site dependencies. In: Golden B, Assad A (eds) Vehicle routing: methods and studies. Elsevier, Amsterdam, pp 149-159

Rapaccini M, Sistemi A, Visintin F (2008) A simulation-based DSS for field service delivery optimization. In: Proceedings of the international workshop on modelling and applied simulation (MAS 2008), 17-19 September 2008, Campora San Giovanni, Amantea (CS), Italy, pp 116-122. ISBN 978-88-903724-1-4

Rardin R, Choe U: Tighter relaxations of fixed charge network flow problems, Technical Report J-79-18, Georgia Institute of Technology, Atlanta, Georgia (1979) 